\title{
Advances in Post-necking Flow Curve Identification of Sheet Metal through Standard Tensile Testing
}

\author{
Sam Coppieters*, ${ }^{* \dagger}$ Steven Cooreman**, Dimitri Debruyne*,‡ and Toshihiko \\ Kuwabara $^{\dagger}$ \\ * Department of Mechanical Engineering, Catholic University College Ghent, Association KU Leuven, \\ Gebroeders De Smetstraat 1, B-9000 Gent, Belgium. \\ ${ }^{\dagger}$ Division of Advanced Mechanical Systems Engineering, Institute of Engineering, Tokyo University of \\ Agriculture and Technology, 2-24-16 Naka-cho, Koganei-shi, Tokyo 184-8588, Japan. \\ ${ }^{* *}$ ArcelorMittal Global R\&D Gent, OCAS NV, Pres. J.F. Kennedylaan 3, 9060 Zelzate, Belgium \\ ${ }^{\ddagger}$ Department of Metallurgy and Materials Engineering, KU Leuven, Kasteelpark Arenberg 44 bus 2450, 3001 \\ Heverlee, Belgium.
}

\begin{abstract}
The standard tensile test is still the most common material test to identify the hardening behavior of sheet metal. When using standard equipment and well-known analytical formulas, however, the hardening behavior can only be identified up to the point of maximum uniform elongation. Several methods which deal with the problem of extended flow curve identification of sheet metal through a tensile test have been proposed in the past. This paper gives an overview of the four classes of methods to identify post-necking hardening behavior of sheet metal through tensile testing. In addition, identification methods from the first (average values across the neck), second (Bridgeman correction, modified Siebel and Schwaigerer correction) and third class (special case of the VFM) are used to identify the post-necking hardening behavior of DC05. Finally, these results are used to assess the validity of the different methods.
\end{abstract}

Keywords: Post-necking hardening behavior, digital image correlation, sheet metal, tensile test.

PACS: 81.40.Ef;81.70.-q;62.20.D-, 81.40.Jj;62.20.F-

\section{INTRODUCTION}

At present, the tensile test is for historical reasons and because it is easy to perform probably the most widely adopted material test for sheet metal. The test allows to identify the hardening behavior up to the point of diffuse necking, at least if standard equipment is used to measure load-elongation data. Many sheet metal forming processes, however, generate strains far beyond the point of maximum uniform strain. With the widespread availability of Digital Image Correlation (DIC) it became possible to measure the strain field inside the diffuse neck. There are currently, however, several ways to use these strain fields to identify the the material behavior hidden in the post-necking regime of a tensile specimen. In the pioneering work of Bridgman [1] three levels of approximation with respect to the problem of diffuse necking in a tensile specimen were recognized. The first class of methods takes necking into account by using average values across the diffuse neck and assuming a uni-axial stress state. Pioneering work of Bridgman resulted in a second, more sophisticated class of methods which are concerned with the distribution of stress and strain across the diffuse neck. The third class consists of methods which use the complete solution of the general problem and consider the material state and the shape of the whole tensile specimen during diffuse necking. The first part of this paper gives an overview of these methods and classifies them by their level of approximation. In addition, an alternative complete solution method is presented which is targeted to avoid the shortcomings of the FE-based inverse method. The second part of the paper embarks on the accuracy of the different levels of approximation. To this purpose, a quasi-static tensile test on a mild steel sheet (DC05) is conducted and the strain fields in the diffuse neck are measured using DIC.

\section{POST-NECKING FLOW CURVE IDENTIFICATION OF SHEET METAL}

The first level of approximation takes diffuse necking into account by averaging strain and stress over the minimum cross section. This means that the stress state is assumed to remain uni-axial in the post-necking regime. The true stress is usually approximated by simply dividing the total tensile load on the specimen by the current minimum cross 
section. The true strain associated with a certain true stress level is approximated by Eq.(1). Where $A_{\min }$ and $A_{0}$ are the minimum cross section and the initial cross section, respectively. Another common method is to extrapolate the pre-necking hardening behavior to high plastic strains using a phenomenological hardening law. The extrapolation method (EM) is considered here as a first approximation which may or may not be valid depending on the a priori chosen hardening law.

$$
\varepsilon_{\text {true }}=\ln \left(\frac{A_{\min }}{A_{0}}\right)
$$

The second level of approximation was defined by Bridgman [1] as the level which tries to take the stress and strain distribution across the diffuse neck into account. Pioneering work on the derivation of the stress distribution in the neck of a cylindrical tensile specimen was performed by Bridgman [1] and Davidenkov [2]. Their mathematical analysis relied on expressing equilibrium across the diffuse neck and provided a correction to the average stress $\sigma_{a v g}$ to compensate for the introduction of transverse stresses. Bridgman derived an analytical solution for flat specimen which read as:

$$
\sigma_{e q}=\frac{\sigma_{a v g}}{\left(1+\frac{4 R}{w}\right)^{\frac{1}{2}} \log \left[1+\frac{w}{2 R}+\left(\left(\frac{w}{R}\right)^{\frac{1}{2}}\right)\left(1+\frac{w}{4 R}\right)^{\frac{1}{2}}\right]-1}
$$

where $\mathrm{w}$ and $\mathrm{R}$ are geometrical parameters in the diffuse neck. The parameters can be obtained by interrupted testing or by measuring these parameters continuously. Siebel and Schwaigerer [3] also proposed a Bridgman-like correction factor for flat specimen:

$$
\sigma=\sigma_{a v g} \frac{4 R}{4 R+w}
$$

A more detailed numerical analysis which takes the strain heterogeneity into account was performed by Ghosh [4] and Ayres et al. [5]. An actual identification procedure based on these early works is recently proposed by Coruk and Karadogan [6]. Zhang et al. [7] used numerical simulations to provide an analytical calculation of the true cross section of a flat specimen. The second level of approximation has definitely contributed to the understanding of postnecking hardening phenomena. The disadvantage, however, is that it is only concerned with the conditions at the cross section of the diffuse neck. Consequently, the shape of the neck and the conditions elsewhere in the plastically deforming tensile specimen are ignored. The third class consists of methods which use the complete solution of the general problem and consider the material state and the shape of the whole tensile specimen during diffuse necking. It was first simultaneously recognized by Koc et al. [8] and Kajberg et al. [9] that a Finite Element-based (FE-based) inverse strategy can be adopted to identify post-necking hardening behavior. Koc et al. proposed a FE-based inverse procedure based on the experimentally measured tensile forces. Kajberg and co-workers presented virtually the same approach, however, using experimental full-field data to feed the inverse procedure. Due to the use of full-field data which incorporates the necking shape, the identified behavior is expected to be more accurate. An alternative method for identifying constitutive behavior of materials is the Virtual Fields Method (VFM) which has been targeted at avoiding the shortcomings of the FE-based inverse method. An excellent book, written by the pioneers of the method, on the theory and applications of the VFM can be found in [10]. The fundamental theory behind the VFM, as the name suggests, is the principle of virtual work which is written with some particular virtual fields. The first application in metal plasticity using actual experimental data was published by Pannier et al. [11]. The purpose was to retrieve the reference parameters of a Voce law with the VFM. Recently Rossi et al. [12] presented a general VFM-based procedure to extract the constitutive parameters of a plasticity model at large plastic strains using 3D displacement fields. The basic equation for quasi-static non-linear VFM reads as:

$$
-\int_{V} \overline{\bar{\sigma}}: \overline{\overline{\varepsilon^{\star}}} d V+\int_{S} \bar{T} \cdot \overline{u^{\star}} d S=0
$$

In this equation $\overline{\bar{\sigma}}$ and $\bar{T}$ are the actual stress tensor and traction vector, respectively. Furthermore, $\overline{u^{\star}}$ is the virtual displacement field from which the virtual strain field $\overline{\overline{\varepsilon^{\star}}}$ is derived. It is important to understand that in this equation $\overline{\bar{\sigma}}$ and $\overline{\overline{\varepsilon^{\star}}}$ are not coupled through constitutive equations. The virtual displacement can be any function as long as 
it is kinematic admissible (KA). As such, according to the framework of the VFM it is allowed to use the actual displacement fields in Eq.(4) since they are by definition KA. The following equation then holds:

$$
W_{i n t}=\int_{V} \overline{\bar{\sigma}}: \overline{\bar{\varepsilon}} d V=\int_{S} \bar{T} \cdot \bar{u} d S=W_{e x t}
$$

where $\overline{\bar{\sigma}}$ and $\bar{T}$ are the actual stress tensor and traction vector, respectively. Note that $\bar{u}$ and $\overline{\bar{\varepsilon}}$ are the actual displacement field and strain field, respectively. Due to the choice of this particular virtual field (the actual field), Eq.(5) takes the form of a energy balance between internal and external work. $\overline{\bar{\sigma}}$ and $\overline{\bar{\varepsilon}}$ are in this particular case related through the constitutive material behavior. Eq.(5) states that the work performed by the external forces equals the internal work. Through the constitutive equations we can relate the strain field $\overline{\bar{\varepsilon}}$ to the stress field $\overline{\bar{\sigma}}=f(\overline{\bar{\varepsilon}}, \mathbf{q})$. The identification of the finite set of unknown parameters $\mathbf{q}$ is based on the minimization a cost function which reads as:

$$
C(\mathbf{q})=\Sigma_{j=1}^{l}\left[-\int_{V} f\left(\overline{\bar{\varepsilon}}_{j}, \mathbf{q}\right): \overline{\bar{\varepsilon}}_{j} d V+\int_{S} \bar{T}_{j} \cdot \bar{u}_{j} d S\right]^{2}=\Sigma_{j=1}^{l}\left[-\left(W_{i n t}\right)_{j}+\left(W_{\text {ext }}\right)_{j}\right]^{2}
$$

where $\overline{\bar{\varepsilon}}$ is the actual deformation field, $f(\overline{\bar{\varepsilon}}, \mathbf{q})$ is the stress associated through the yield surface of the material and 1 the number of measurements. The use of actual fields instead of virtual fields to identify post-necking hardening behavior of sheet metal was presented in [13]. The major consequence of using actual fields instead of virtual fields is expected to be twofold. First, the cost function expressed by eq.(6) is enriched by the presence of virtual fields. Second, the virtual fields are known to be able to deal with noisy experimental data and hence yield a less biased identification. This is especially true for elastic strains, the choice of virtual fields is, however, reported to be less critical in the case of large plastic strains [10].

\section{EXPERIMENTS AND DISCUSSION OF THE RESULTS}

From the first class the Extrapolation Method (EM) and two simplified methods to measure the strain (Eq.(1)) are used to provide a first approximation of the problem. The latter two methods are referred to as central point neck and average across neck, respectively. Central point neck approximates the equivalent plastic strain by taking the longitudinal strain of the central point in the diffuse neck. For Central point neck the equivalent plastic strain is calculated by taking the average longitudinal strain across the diffuse neck. From the second class the Bridgman correction Eq.(2) and the modified Siebel and Schwaigerer Eq.(3) correction are used to provide a second approximation. Finally, from the third class the special case of the nonlinear VFM presented in the previous section is used to provide a so called Complete Solution (CS). All methods are applied to the data generated by a tensile test in the transverse direction on DC05 reported in [13]. The yield surface is described by Hill's 1948 yield criterion of which the parameters were determined in advance based on the Lankford ratio's in the RD, TD and the $45^{\circ}$-direction. The EM and the CS are used to identify three hardening laws: the Voce law, the Swift law and a weighted combination of the saturating Voce law and the non-saturating Swift law which is referred to as CSV (Eq.7) in the remainder of this paper:

$$
\sigma_{e q}=c\left[K\left(\varepsilon_{0}+\varepsilon_{e q}^{p l}\right)^{n}\right]+(1-c)\left[C\left(1-m e^{-k \varepsilon_{e q}^{p l}}\right)\right]
$$

The identified hardening behavior is shown in figure 1 and it can be inferred that the EM yields very different results depending on the hardening law. For all identified phenomenological hardening laws, the EM Swift law and the EM Voce law seem to predict an upper and a lower bound, respectively. The combined hardening law EM CSV predicts an average behavior. It can be inferred that there is a significant difference between the hardening laws identified by the EM and the CS. Clearly, the complete solution (CS) results in more consistent results since the hardening laws are forced to predict the same behavior. It must be noted that the EM CSV law yields an excellent agreement with CS CSV law up to $2 \varepsilon_{u, \text { max }} \approx 0.46$. From the previous it can be concluded that the CS CSV law is a quite accurate law to describe the hardening behavior of DC05. Therefore the CS CSV curve is used as a reference hardening curve in the discussion of the other methods. If the strain is taken from the central point of the necking zone or averaged across the neck, the hardening curve is in close agreement with the CS CSV law up to $1.5 \varepsilon_{u, \max }$. This suggests that the strain gradient across the width of the specimen can be safely ignored up to this point. Beyond this point, however, very different values are found for both first order approximations indicating that the strain gradients can no longer be ignored. Nevertheless, the remarkable observation here is that the first approximation yields very good results 
up to almost $1.5 \varepsilon_{u, \max }$. This result suggests that the development of transverse stresses is delayed and the uni-axial stress state prevails up to this point well-beyond the maximum load. In this regard, recent work of Iadicola [14] is very interesting since it allows to experimentally validate the stress state in the diffuse necking zone. The conclusions drawn by Iadicola are quantitatively in line with the present study. The overestimation of the equivalent yield stress found by the first approximation should vanish by using the second approximation. As can be inferred from figure 1, both correction methods (Eq.(2) and Eq.(3)) correct the first approximations by lowering the average equivalent stress. The corrected curves, however, remain far from the reference curve (CS CSV law) found by the complete solution. Therefore, it can be concluded that these correction methods are inaccurate for DC05. In conclusion, the first approximations yield good results up to $1.5 \varepsilon_{u, \max }$ for DC05. Beyond this point, the first and second approximations are inaccurate. Today, the strain field inside the neck can be easily measured using DIC. There is, however, no general rule available to determine the point up until the first approximations can be safely applied. Moreover, the level of strain heterogeneity inside the diffuse neck will be material dependent. As such, currently the only way to accurately determine the strain hardening behavior of sheet metal within the diffuse neck is to resort to a complete solution of the problem.

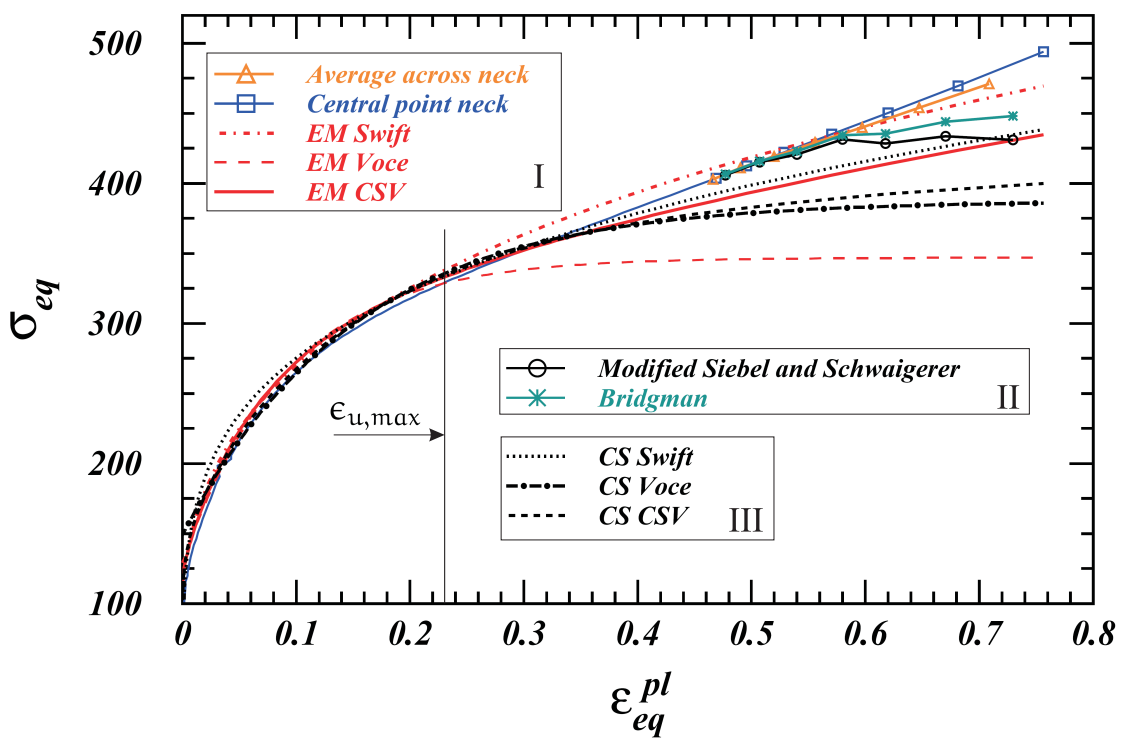

FIGURE 1. Identified pre-necking and post-necking hardening behavior of DC05 through selected methods from the first Class (I), second class (II) and third class (III).

\section{REFERENCES}

1. P.W. Bridgman, Studies in Large Plastic Flow and Fracture. McGraw-Hill, NY, 1952.

2. N.N., Davidenkov, N.I., Spiridonova, Proceedings of ASTM, 46, 1147-1158 (1946).

3. E. Siebel, S. Schwaigerer, Archiv für das Eisenhüttenwesen, 19 (1948).

4. A.K. Ghosh, Metallurgical transactions A, 8A 1221-1232 (1977).

5. R.A. Ayres, Metallurgical transactions A, 14A 2269-2275 (1983).

6. E. Coruk, C. Karadogan, Steel Research International, Special Edition (2011).

7. Z.L. Zhang, M. Hauge, J. Odegard, C. Thaulow, International Journal of Solids and Structures, $363497-3516$ (1999).

8. P. Koc, B. Štok, Computational Materials Science, 31 155-168 (2004).

9. J., Kajberg, G. Lindkvist, International Journal of Solids and Structures, 41 3439-3459 (2004).

10. F. Pierron, M. Grédiac, The Virtual Fields Method. Extracting Constitutive behaviour from full-field deformation measurements. Springer, London, 2012.

11. Y. Pannier, S. Avril, R. Rotinat, F. Pierron, Experimental Mechanics, 46 735-755 (2006).

12. M. Rossi, F. Pierron, Computational Mechanics, 59 53-71 (2011).

13. S. Coppieters, S. Cooreman, H. Sol, P. Van Houtte, D. Debruyne, Journal of Materials Processing Technology, 211 545-552 (2011).

14. M.A. Iadicola, AIP conference proceedings, 1383, $742-749$ (2011). 